\title{
1-YIA-18 YIA
}

\section{Rapid measurement of plasma concentration of a molecular-targeted agent, pazopanib, with diamond sensor.}

\author{
Saiki Takuro ${ }^{1,2}$, Genki Ogata ${ }^{1}$, Rito Kato ${ }^{1}$, Olga Razvina ${ }^{1}$, Seishiro Sawamura ${ }^{1}$, \\ Yoshifumi Matsumoto ${ }^{2}$, Ai Hanawa ${ }^{3}$, Kai Asai ${ }^{3}$, Yasuaki Einaga ${ }^{3}$, Yasuo Saijo ${ }^{2}$, Hiroshi Hibino ${ }^{1}$
}

${ }^{I}$ Dept Mol Physiol, Niigata Univ Sch Med, ${ }^{2}$ Dept Med Oncol, Niigata Univ Sch Med, ${ }^{3}$ Dept of Chem, Fac of Sci and Tech, Keio Univ

Molecular-targeted anticancer drugs elicit less toxicity than conventional reagents. Yet, patients often suffer from severe adverse effects. A reason is 'fixed dosage' administration of the drug to all the patients regardless of their body size and complications; because of this strategy, the plasma concentration seems to exceed the therapeutic window occasionally. Although frequent measurement of the drug level at a clinical site is a solution, currently available methods such as mass spectrometry are time and cost consuming. To overcome these shortcomings, in this study, we developed a procedure with an electrochemical sensor composed of a conductive diamond, which yields more stable reactions than conventional materials. When guinea-pig plasma mixed with pazopanib, a multi-kinase inhibitor, was tested, the sensor detected a clinically relevant concentration of 3 to $300 \mu \mathrm{M}$. Time and sample amount necessary for each series of the measurement was $<1$ min and $100 \mu \mathrm{L}$, respectively. The sensor was repeatedly usable with minimal impairment of the sensitivity, saving the cost for the assay. This rapid and easily-handed method may enable therapeutic drug monitoring and accelerate tailored medicine for cancer. 